OPEN ACCESS

Edited by: Carsten Watzl,

Leibniz Research Centre for Working

Environment and Human Factors

(LG), Germany

Reviewed by:

Francisco Borrego,

BioCruces Health Research Institute,

Spain

Francesco Colucci,

University of Cambridge,

United Kingdom

*Correspondence:

Quirin Hammer

quirin.hammer@drfz.de

Specialty section:

This article was submitted to NK and Innate Lymphoid

Cell Biology,

a section of the journal

Frontiers in Immunology

Received: 29 October 2017 Accepted: 20 December 2017

Published: 17 January 2018

Citation:

Hammer Q, Rückert T, Dunst J and Romagnani C (2018) Adaptive

Natural Killer Cells Integrate Interleukin-18 during Target-Cell

Encounter.

Front. Immunol. 8:1976. doi: 10.3389/fimmu.2017.01976

\section{Adaptive Natural Killer Cells Integrate Interleukin-18 during Target-Cell Encounter}

\author{
Quirin Hammer ${ }^{1 *}$, Timo Rückert ${ }^{1}$, Josefine Dunst ${ }^{2}$ and Chiara Romagnani ${ }^{1,3}$
}

${ }^{1}$ Innate Immunity, German Rheumatism Research Center (DRFZ), Leibniz Association, Berlin, Germany, ${ }^{2}$ Inflammation Biology, German Rheumatism Research Center (DRFZ), Leibniz Association, Berlin, Germany, ${ }^{3}$ Medical Department I, Charité - University Medicine Berlin, Berlin, Germany

Human cytomegalovirus (HCMV) infection induces adaptations in the natural killer (NK)-cell compartment. Expanded subsets of adaptive NK cells display potent effector functions against cellular targets, despite their apparent unresponsiveness to stimulation with classical dendritic cell-derived cytokines interleukin (IL)-12 and IL-18. However, it remains unclear whether adaptive NK cells have completely lost their ability to sense inflammation via IL-12 and IL-18 or whether these pro-inflammatory signals can be functionally integrated into defined contexts. Here, we demonstrate that adaptive NKG2C+ NK cells can be costimulated by the presence of pro-inflammatory cytokines during target cell-induced activation. Cytokine costimulation of adaptive NK cells resulted in elevated interferon (IFN)-gamma and tumor necrosis factor (TNF) production, which promoted protein expression of HLA class I and adhesion molecules as well as transcription of genes involved in antigen processing and antiviral states in endothelial bystander cells in vitro. We further show that IL-18 drove costimulation in functional assays and was sufficient for elevated cytokine production in the absence of IL-12. Hence, adaptive NKG2C+ $\mathrm{C}^{+}$KK cells - although poorly responsive to IL-12 and IL-18 as an isolated stimulus -integrate IL-18 as a costimulatory signal during target-cell encounter.

Keywords: human natural killer cells, adaptive natural killer cells, NKG2C, interleukin-18, costimulation

\section{INTRODUCTION}

Natural killer (NK) cells are lymphocytes required for proficient immunity against viral infections (1), and as members of the innate lymphoid cell family, NK cells are classically regarded part of the innate immune system (2). However, emerging experimental evidence suggest that NK cells can display adaptive-like features in response to inflammatory signals $(3,4)$, hapten challenge (5), and especially viral infection $(6,7)$.

In humans, adaptive NK-cell responses are driven by human cytomegalovirus (HCMV) infection and are associated with the expansion of subsets expressing the activating receptor NKG2C (8). Adaptive $\mathrm{NKG}_{2} \mathrm{C}^{+} \mathrm{NK}$ cells in HCMV-seropositive individuals display skewed and narrow expression patterns of otherwise stochastically distributed killer Ig-like receptors, which is suggestive of oligoclonal or clonal-like expansion upon HCMV infection and parallels the pathogen-specific responses of $\mathrm{T}$ cells $(9,10)$. Furthermore, HCMV-induced adaptive NK cells display a remodeled epigenetic landscape analogous to the global differentiation program of effector-memory CD8 ${ }^{+}$ T cells $(11,12)$. The loss of DNA methylation at regulatory regions within the TNF and IFNG loci is 
peculiarly shared between adaptive NK cells and terminally differentiated $\mathrm{T}$ cells $(11,12)$, enabling robust cytokine production and highlighting adaptive traits at the molecular level.

The functionality of adaptive NK cells is further calibrated by their activating receptor expression pattern, which determines their recognition properties [reviewed in Ref. (13)]. Adaptive NK cells largely lack natural cytotoxicity receptors such as NKp30 and $\mathrm{NKp} 46$, but preferentially express the activating receptor $\mathrm{NKG} 2 \mathrm{C}$ and the costimulatory receptor $\mathrm{CD} 2$, while other activating receptors such as CD16 are similarly expressed by adaptive and conventional NK cells $(8,10,14)$. Accordingly, adaptive NK cells proficiently produce cytokines upon engagement of NKG2C or CD16 by HLA-E-expressing or antibody-coated target cells, respectively (9), and cross-linking of CD2 can further amplify adaptive NK-cell functions (14). In contrast to conventional NK cells, adaptive NK cells were reported to display poor responsiveness toward the classical NK cell-activating dendritic cell-derived cytokines, interleukin (IL)-12 and IL-18 (9, 12), suggesting an altered recognition strategy poised for responses against defined cellular targets.

However, both infected cells and a robust inflammatory milieu are present during viral infection (15-17), and it remains incompletely understood whether adaptive $\mathrm{NKG}_{2} \mathrm{C}^{+} \mathrm{NK}$ cells have completely lost their ability to sense IL-12 and IL-18 (IL-12 + 18) and rely solely on recognition of cellular stimuli, or whether adaptive $\mathrm{NKG}_{2} \mathrm{C}^{+} \mathrm{NK}$ cells are able to functionally respond to these inflammatory cues in the context of target-cell encounter.

Here, we show that adaptive $\mathrm{NKG}_{2} \mathrm{C}^{+} \mathrm{NK}$ cells are poorly responsive to $\mathrm{IL}-12+18$ as a single stimulus, but if provided alongside target cells, IL-12 + 18 results in amplification of adaptive $\mathrm{NKG}_{2} \mathrm{C}^{+} \mathrm{NK}$-cell cytokine production. We further demonstrate that cytokine costimulated adaptive $\mathrm{NKG} 2 \mathrm{C}^{+} \mathrm{NK}$ cells relay enhanced activation to bystander cells and that IL- 18 functionally drives elevated cytokine production during target-cell encounter.

\section{RESULTS}

\section{Effector Responses of Adaptive NK Cells against Target Cells Are Amplified by Cytokine Costimulation}

Reprogrammed effector functions are a hallmark of adaptive NK cells and, in line with previous data $(9,12)$, only a minor fraction of adaptive $\mathrm{NKG} 2 \mathrm{C}^{+} \mathrm{NK}$ cells produced the NK-cell signature cytokine interferon (IFN)- $\gamma$ after $24 \mathrm{~h}$ stimulation with IL- $12+18$ as compared to conventional NKG2 $\mathrm{C}^{-} \mathrm{NK}$ cells (Figures 1A,B), suggesting that adaptive NK cells are largely insensitive to these pro-inflammatory cytokines as a single stimulus.

To test whether adaptive NK cells have retained the capacity to integrate IL- $12+18$ as a costimulatory signal during targetcell encounter, we cultured FACS-purified NK cells with target cells in the absence or presence of IL-12 + 18. Adaptive NKG2C ${ }^{+}$ NK cells proficiently produced IFN- $\gamma$ and tumor necrosis factor (TNF) upon stimulation with K562/HLA-E cells, which express HLA-E and engage the key activating receptor NKG2C (Figures 1C,G). Importantly, the presence of IL-12 + 18 during target-cell stimulation consistently increased the frequency of IFN- $\gamma^{+}$as well as $\mathrm{TNF}^{+}$adaptive NK cells (Figures $\mathbf{1 D}, \mathbf{H}$ ), indicating that these pro-inflammatory cytokines can still function as costimulatory signals for adaptive NK cells. Moreover, the addition of IL-12 +18 resulted in elevated signal intensity of both IFN- $\gamma$ and TNF (Figures 1E,I), and presence of IL-12 + 18 during target-cell encounter was associated with significantly heightened IFN- $\gamma$ and TNF protein concentrations in the supernatant of cocultures (Figures 1F,J), thus further corroborating a costimulatory effect.

Increased frequencies of IFN $-\gamma^{+}$and $\mathrm{TNF}^{+}$adaptive NK cells were also detected after culture with beads coated with an agonistic anti-NKG2C antibody in the presence of IL-12 + 18 (Figures S1D,E in Supplementary Material), suggesting that sole ligation of NKG2C is sufficient to enable costimulatory effects of IL-12 +18 .

Furthermore, elevated IFN- $\gamma$ and TNF production was similarly observed after culture with rituximab-coated 721.221 B-cell lymphoma cells in the presence of IL-12 + 18 (Figures $\mathrm{S} 1 \mathrm{~F}, \mathrm{H}$ in Supplementary Material), implying that potentiation of cytokine production by pro-inflammatory signals is not restricted to NKG2C-mediated activation but also detectable upon engagement of other adaptive NK-cell activating receptors such as CD16.

Altogether, these data demonstrate that-although largely unresponsive to IL-12 +18 as an isolated stimulus-adaptive NK cells can integrate IL- $12+18$ into effector responses against target cells, resulting in augmented cytokine production.

\section{Cytokine Costimulated Adaptive NK Cells Proficiently Alert Bystander Cells In Vitro}

Natural killer cell-derived IFN- $\gamma$ and TNF mediate pleiotropic effects on both immune and non-immune bystander cells such as endothelial cells, which can be alerted to participate in immune responses as conditional antigen-presenting cells due to their ability to present antigen and regulate influx of antigen-specific lymphocytes via adhesion molecules (18-21). To test the functional capacity of IL-12+18 costimulated adaptive NK cells and to investigate whether the integration of pro-inflammatory signals during target-cell recognition can be relayed to bystander cells, human umbilical vein endothelial cells (HUVEC) were treated with conditioned medium obtained from supernatants of FACSsorted adaptive $\mathrm{NKG}_{2} \mathrm{C}^{+} \mathrm{NK}$ cells cocultured with K562/HLA-E either in the absence or presence of IL-12 + 18 (Figure 2A). In line with the reported contribution of IFN- $\gamma$ and TNF in activating endothelial cells $(18,21)$, medium conditioned by K562/HLA-Estimulated adaptive NK cells induced clear upregulation of HLA class I protein on HUVEC (Figure 2B). Importantly, HUVEC responded to conditioned medium from IL-12 + 18 costimulated adaptive NK cells with consistently higher HLA class I expression (Figure 2B) while addition of IL-12 + 18 directly to HUVEC had no effect (Figure S2A in Supplementary Material), suggesting that increased cytokine output resulting from IL-12 + 18 costimulation of adaptive $\mathrm{NKG}_{2} \mathrm{C}^{+} \mathrm{NK}$ cells can be relayed to bystander cells.

Increase of HLA class I protein levels was blocked upon addition of anti-IFN- $\gamma$ and anti-TNF antibodies to HUVEC, indicating that NK cell-secreted IFN- $\gamma$ and TNF played major roles in 
A

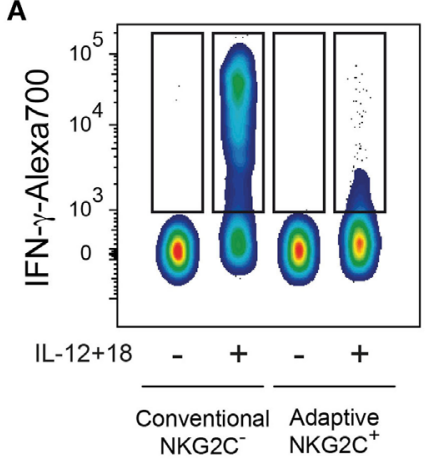

C

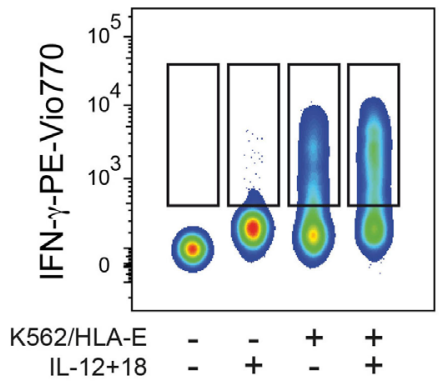

G

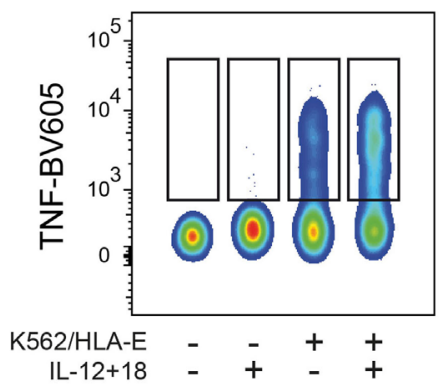

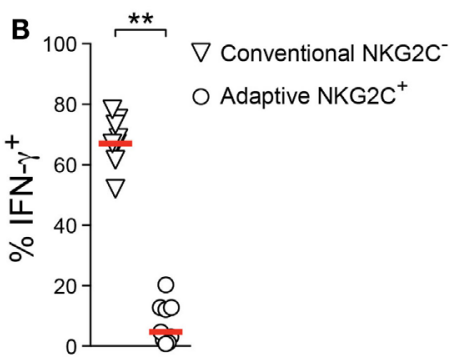

D
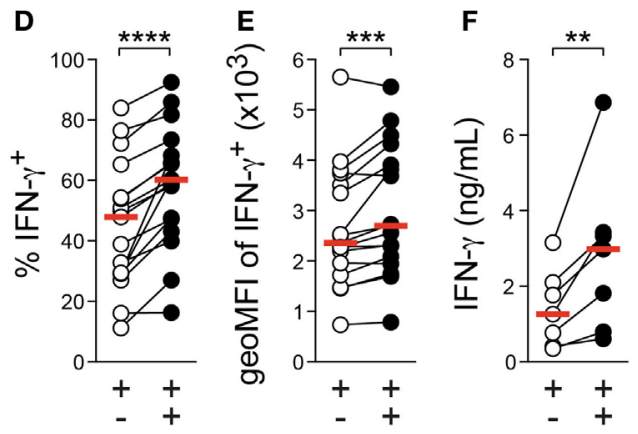

H

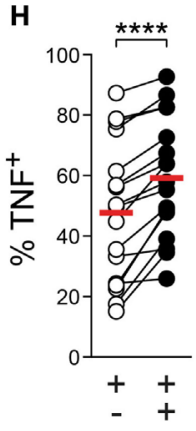

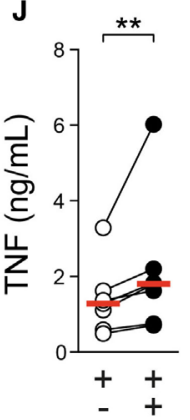

FIGURE 1 | Effector responses of adaptive natural killer (NK) cells against target cells are amplified by cytokine costimulation. (A) Representative staining of interferon (IFN)- $\gamma$ gated on conventional NKG2C- or adaptive NKG2C+ NK cells after 24 h culture in the absence or presence of interleukin (IL)-12 + 18. (B) Summary of frequencies of IFN- $\gamma^{+}$cells. Symbols indicate individual donors, and red lines indicate median $(n=9)$. (C) Representative staining of IFN- $\gamma$ gated on adaptive NKG2C+ NK cells after 6 h culture either alone or with K562/HLA-E target cells in the absence or presence of IL-12 + 18. (D) Summary of frequencies of IFN- $\gamma^{+}$cells and (E) summary of geometric mean fluorescence intensity (geoMFI) of IFN- $\gamma$ in IFN- $\gamma^{+}$cells, $n=17$ donors. (F) Summary of IFN- $\gamma$ protein concentrations in the supernatant of cocultures, $n=7$ donors. (G) Representative staining of tumor necrosis factor (TNF) gated on adaptive NKG2C+ NK cells after $6 \mathrm{~h}$ culture either alone or with K562/HLA-E target cells in the absence or presence of IL-12 + 18. (H) Summary of frequencies of TNF+ cells and (I) summary of geoMFI of TNF in TNF+ cells, $n=17$ donors. (J) Summary of TNF protein concentrations in the supernatant of cocultures, $n=7$ donors. Connected symbols indicate individual donors, and red lines indicate median. All statistical analyses performed with one-tailed Wilcoxon matched-pairs test. ${ }^{\star} p<0.05$, ${ }^{\star \star} p<0.01$, ${ }^{\star \star \star} p<0.001$, and ${ }^{* \star \star \star} p<0.0001$.

mediating endothelial-cell activation (Figure 2B; Figure S2B in Supplementary Material).

Furthermore, TNF-dependent induction of E-selectin surface expression was consistently elevated after incubation with conditioned medium from adaptive NK cells costimulated by IL-12 + 18 (Figure 2C; Figure S2C in Supplementary Material). HUVEC incubated with conditioned medium of stimulated adaptive NK cells homogenously expressed ICAM-1 at the cell surface, and its expression levels were mildly increased when adaptive NK cells were costimulated with exogenous cytokines (Figure S2D in Supplementary Material), while VCAM-1 was not preferentially affected (Figure S2E in Supplementary Material).

In agreement with endothelial-cell activation, transcripts of $B 2 M$ (encoding the $\beta_{2}$ microglobulin component of HLA class I heterodimers) and PSMB9 (encoding the inducible immunoproteasome subunit $\beta 9$ ) were induced in HUVEC treated with medium conditioned by K562/HLA-E-activated adaptive 


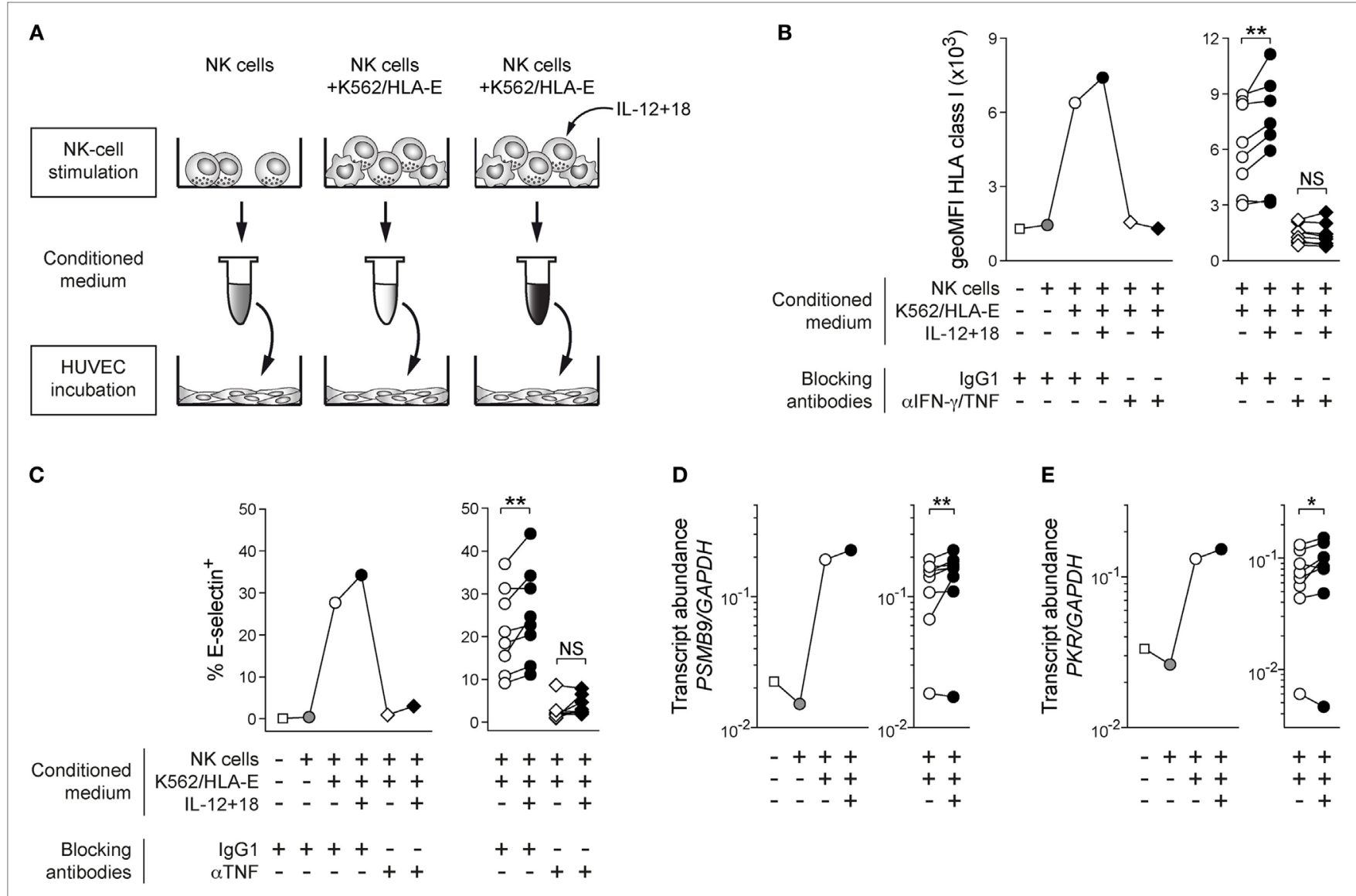

FIGURE 2 | Cytokine costimulated adaptive natural killer (NK) cells proficiently alert bystander cells in vitro. (A) Schematic illustration of experimental design. (B) Representative geoMFI of HLA class I on human umbilical vein endothelial cells (HUVEC) after $40 \mathrm{~h}$ treatment with indicated conditioned medium in the presence or absence of anti-interferon (IFN)- $\gamma$ and anti-tumor necrosis factor (TNF) blocking antibodies (left) and summary of $n=8$ treatments with conditioned medium from independent NK-cell stimulations (right). (C) Representative frequency of E-selectin+ $\mathrm{HUVEC}$ after $6 \mathrm{~h}$ treatment with indicated conditioned medium in the presence or absence of anti-TNF blocking antibodies (left) and summary of $n=8$ treatments with conditioned medium from independent NK-cell stimulations (right). (D) Representative PSMB9 transcript abundance relative to GAPDH in HUVEC after $24 \mathrm{~h}$ treatment with indicated conditioned medium (left) and summary of $n=8$ NK-cell stimulations (right). (E) Representative protein kinase R (PRK) PKR transcript abundance relative to GAPDH in HUVEC after $24 \mathrm{~h}$ treatment with indicated conditioned medium (left) and summary of $n=8$ treatments with conditioned medium from independent NK-cell stimulations (right). Connected symbols indicate individual donors used to generate conditioned medium. All statistical analyses performed with one-tailed Wilcoxon matched-pairs test. NS, not significant; ${ }^{*} p<0.05$ and ${ }^{* *} p<0.01$.

NKG2C ${ }^{+} \mathrm{NK}$ cells and further upregulated when IL-12 + 18 was present during NK-cell stimulation (Figure 2D; Figure S2F in Supplementary Material), indicating that transcription of genes involved in both antigen presentation and antigen processing was preferentially triggered in HUVEC when adaptive NK cells integrated inflammatory signals during target-cell encounter.

Additionally, we observed that expression of the inducible antiviral effector molecule protein kinase R [PKR; (18)] was similarly elevated at the mRNA level (Figure 2E), implying that IL-12 + 18-mediated costimulation of adaptive $\mathrm{NKG}_{2} \mathrm{C}^{+} \mathrm{NK}$ cells can efficiently prime bystander cells to acquire antiviral states.

Thus, these data suggest that the presence of IL- $12+18$ during target-cell encounter has functional consequences and enables adaptive $\mathrm{NKG}_{2} \mathrm{C}^{+} \mathrm{NK}$ cells to proficiently alert bystander endothelial cells by instructing enhanced protein expression of HLA class I and adhesion molecules as well as promote transcription of genes involved in antigen processing and antiviral functions.

\section{IL-18 Drives Costimulation of Adaptive NK Cells}

Since our data point toward a merely costimulatory function of IL-12 + 18 for adaptive NK cells, we sought to better understand the underlying regulation. We therefore analyzed the transcript expression of IL12RB2 and IL18RAP in ex vivo FACS-sorted terminally differentiated adaptive $\mathrm{NKG}^{2} \mathrm{C}^{+} \mathrm{NK}$ cells (CD56 $6^{\mathrm{dim}}$ $\mathrm{CD}^{2} 7^{+} \mathrm{NKG}_{2} \mathrm{C}^{+}$), mature conventional $\mathrm{NK}$ cells (CD56 $6^{\mathrm{dim}}$ $\mathrm{CD} 7^{+} \mathrm{NKG}^{-} \mathrm{C}^{-}$), and immature conventional NK cells (CD56 $6^{\mathrm{dim}}$ $\left.\mathrm{CD}^{-} 7^{-} \mathrm{NKG}^{-} \mathrm{C}^{-}\right)$together with naive $\left(\mathrm{CD} 45 \mathrm{RA}^{+} \mathrm{CD}^{+} 5 \mathrm{RO}^{-}\right.$ $\left.\mathrm{CCR7}^{+}\right)$and effector-memory $\left(\mathrm{CD}^{-} 5 \mathrm{RA}^{-} \mathrm{CD}^{-} 5 \mathrm{RO}^{+} \mathrm{CCR}^{-}\right)$ $\mathrm{CD} 8^{+} \mathrm{T}$ cells (Figures $\mathrm{S} 3 \mathrm{~A}, \mathrm{~B}$ in Supplementary Material). In 
this setting, naïve $\mathrm{CD} 8^{+} \mathrm{T}$ cells serve as a negative control due to their unresponsiveness to IL- $12+18$, while effector-memory $\mathrm{CD}^{+} \mathrm{T}$ cells profoundly produce IFN- $\gamma$ upon stimulation with IL-12 + 18 in vitro and in vivo (22-24). In line with previous data (12), IL12RB2 transcripts were clearly reduced in adaptive $\mathrm{NKG}_{2} \mathrm{C}^{+} \mathrm{NK}$ cells compared to immature NK cells (41-fold decrease in median transcript abundance), approaching the expression level of naïve $\mathrm{CD}^{+} \mathrm{T}$ cells (Figure 3A). IL18RAP mRNA was also reduced in adaptive $\mathrm{NKG}_{2} \mathrm{C}^{+}$when compared to immature NK cells (Figure 3B); however, this reduction was modest (2.2-fold decrease in median transcript abundance) and IL18RAP transcript levels of adaptive $\mathrm{NKG}^{2} \mathrm{C}^{+} \mathrm{NK}$ cells were not significantly different from those of effector-memory $\mathrm{CD}^{+}$ T cells (Figure 3B). Furthermore, diminished IL12RB2 transcript levels correlated with those of ZBTB16 (encoding PLZF), while this was not the case for IL18RAP (Figure S3C in Supplementary Material), suggesting an altered cytokine receptor profile of adaptive NK cells, which might result in a selective unresponsiveness toward IL-12 and potentially involves distinct regulatory programs controlling the disparate expression of cytokine receptors.

In agreement with previous reports, IL-12 failed to transmit signals via phosphorylated STAT4, while treatment with IL-18 resulted in consistent phosphorylation of NF- $\kappa \mathrm{B}$ in FACS-sorted adaptive NK cells [(12); Figures 3C,D; Figures S3D-G in Supplementary Material], implying that IL-18 could contribute to coactivate adaptive NK-cell effector responses.

To ascertain whether IL-18 is the decisive signal for amplifying cytokine responses, we dissected the individual contributions of IL-12 and IL-18 during target-cell encounter. Indeed, IL-18 drove costimulation of adaptive NK cells in functional assays and was sufficient to augment IFN- $\gamma$ and TNF production during coculture with K562/HLA-E target cells or $\alpha$ NKG2C beads, while IL-12 did not impact on the frequencies of cytokine-producing cells (Figures 3E,F; Figures S3G,H in Supplementary Material). Functional responses of adaptive NK cells to IL-18 costimulation reached a plateau at $10 \mathrm{ng} / \mathrm{mL}$ (Figures 3G,H), suggesting that low doses of IL-18 can potentiate adaptive NK-cell activation. Conversely, addition of IL-12 to functional assays did not impact on adaptive NK-cell cytokine production, nor did IL-12 synergize with IL-18 for costimulation (Figures 3E,F; Figures S3G,H in Supplementary Material).

Collectively, these data demonstrate that the presence of pro-inflammatory cytokines during target-cell encounter results in amplified IFN- $\gamma$ and TNF production by adaptive NK cells. The amplified activation can be relayed to bystander endothelial cells, which in turn acquire elevated antigen processing and presentation capacity as well as antiviral states. Selective responsiveness toward IL-18 but not IL-12 for costimulation of target cell-induced activation highlights the refined receptor expression pattern of adaptive NK cells and further emphasizes their restricted recognition strategy, which enables adaptive NK cells to proficiently respond to finely selected stimuli.

\section{DISCUSSION}

Adaptive $\mathrm{NKG}_{2} \mathrm{C}^{+} \mathrm{NK}$ cells are potent effectors against HLAE-expressing and antibody-coated target cells while considered insensitive to innate cues such as the pro-inflammatory cytokines IL-12 and IL-18. Yet, it is not known whether adaptive NK cells have completely lost the responsiveness to IL-12 and IL-18 or whether they can respond to inflammatory signals in a specific context. Here, we demonstrate that IL-18 can potentiate adaptive $\mathrm{NKG}_{2} \mathrm{C}^{+} \mathrm{NK}$-cell activation elicited by target cells. The presence of IL-18 during target-cell encounter augments adaptive NK-cell cytokine production, likely by phosphorylation of NF- $\mathrm{KB}$, which potentially synergizes with NKG2C- or CD16-mediated signaling for optimal IFN- $\gamma$ and TNF release.

Interestingly, murine adaptive $\mathrm{Ly} 49 \mathrm{H}^{+} \mathrm{NK}$ cells arising in response to MCMV infection (6) are strictly dependent on IL-12 for their generation (25). However, when fully differentiated, murine adaptive $\mathrm{Ly}_{4} 9 \mathrm{H}^{+} \mathrm{NK}$ cells respond poorly to cytokinemediated activation caused by Listeria or influenza infection in vivo, and their responsiveness to IL-12 in vitro is drastically diminished, although not completely abolished (26). Indeed, residual STAT4 phosphorylation is detectable and cross-linking of Ly49H can be costimulated by IL-12 in vitro (26). In contrast, likely due to drastic downregulation of IL12RB2 transcripts, human adaptive $\mathrm{NKG}_{2} \mathrm{C}^{+} \mathrm{NK}$ cells have fully lost their ability to respond to IL-12: IL-12 does not induce phosphorylation of STAT4 nor promote elevated IFN- $\gamma$ or TNF production during target-cell encounter. Conversely, IL18RAP transcript levels are sustained in adaptive $\mathrm{NKG} 2 \mathrm{C}^{+} \mathrm{NK}$ cells and, although phosphorylation of NF- $\kappa \mathrm{B}$ is less efficient in adaptive compared to conventional NK cells, IL-18 is preserved as a functional costimulatory signal for adaptive NK-cell effector responses. The disparate expression levels of IL12RB2 and IL18RAP parallel and extend the previously described skewed receptor pattern of adaptive NK cells [reviewed in Ref. (13)]: adaptive NK cells express reduced frequencies of activating receptors such as NKp30 or NKp46 compared to conventional NK cells, while maintaining CD16 and preferentially expressing NKG2C, which restrains the recognition properties of adaptive NK cells. Consequently, the restricted recognition repertoire renders adaptive NK cells especially responsive to specific stimuli such as HLA-E-expressing or antibody-coated targets, while cells expressing ligands for NKp30 or NKp46 inadequately trigger adaptive NK-cell functions. Similarly, IL-18 costimulation results in amplified cytokine production, whereas IL-12 has no functional impact.

Selective sensing of IL-18 but not IL-12 by human adaptive NK cells is of particular interest since multiple infections and inflammatory diseases are associated with systemic or local cytokine induction $(27,28)$. In this context, unresponsiveness of HCMV-induced adaptive NK cells to IL-12 might guard the population against IL-12-mediated activation-induced cell death during unrelated infections (29), thereby contributing to long-term maintenance of adaptive NK cells $(30,31)$. Intriguingly, IL- 18 is a central component of the global cytokine signature detected in patients experiencing HCMV reactivation (32), which suggests that HCMV-infected host cells and systemic IL-18 are concomitantly present during acute infection. The presence of varying IL-18 levels during target-cell encounter potentially fine-tunes cytokine secretion of adaptive $\mathrm{NKG}_{2} \mathrm{C}^{+} \mathrm{NK}$ cells and enables optimal effector functions. 

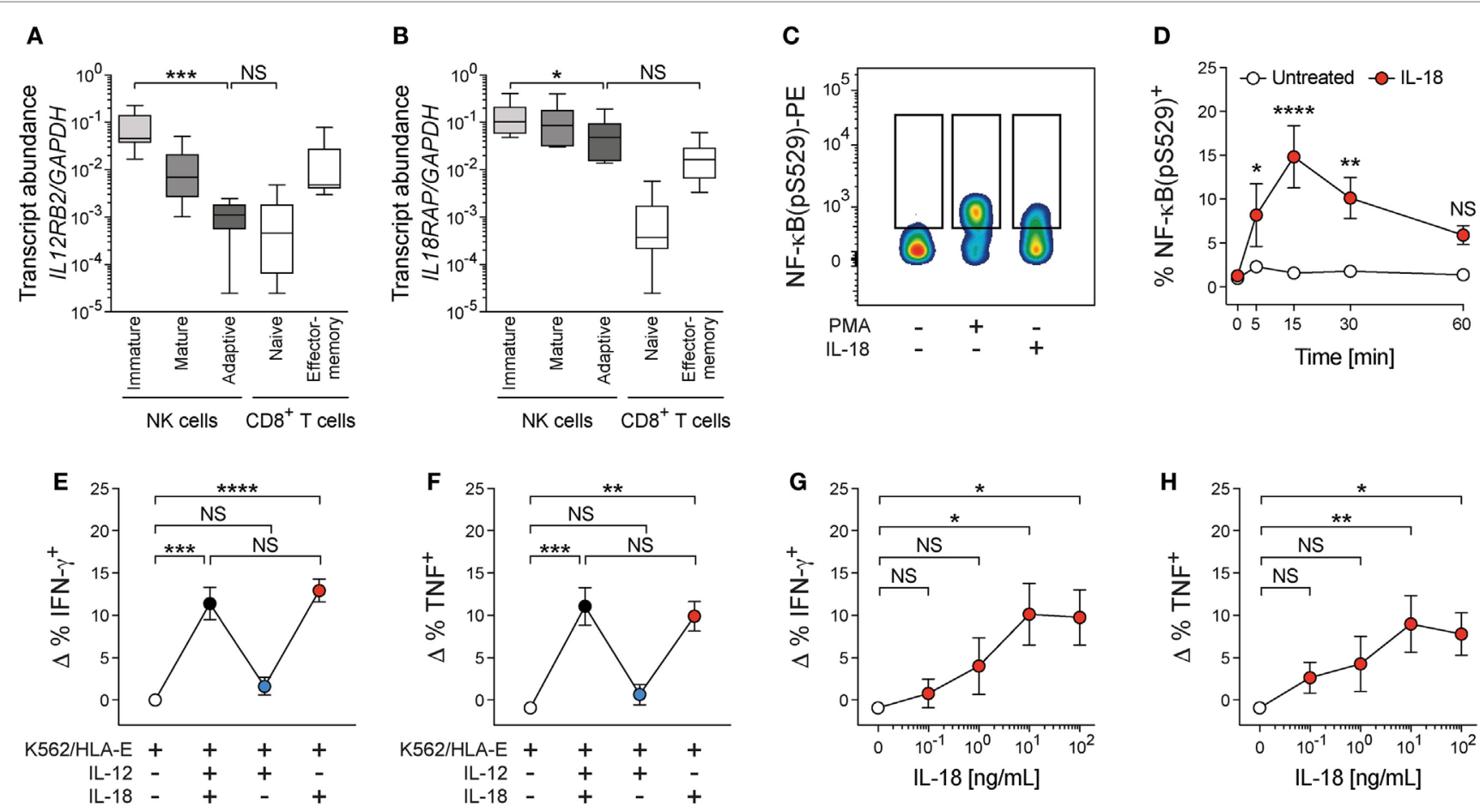

FIGURE 3 | Interleukin (IL)-18 drives costimulation of adaptive natural killer (NK) cells. (A) IL12RB2 and (B) IL18RAP transcript abundance relative to GAPDH in ex vivo FACS-purified immature conventional NK cells (CD56 dim CD57- NKG2C-), mature conventional NK cells (CD56 dim CD57 ${ }^{+} \mathrm{NKG}^{\text {di }} \mathrm{C}^{-}$), adaptive NKG2C+

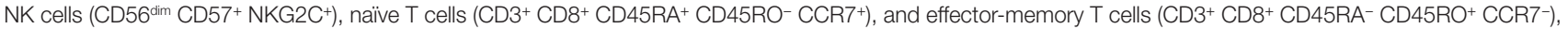
$n=6$ individual donors. Box plots display minimum to maximum and median. Statistical analysis performed with Friedman and Dunn's multiple comparison test. (C) Representative staining of NF-kB(pS529) in FACS-purified adaptive NKG2C+ NK cells after treatment with medium, phorbol-12-myristat-13-acetat (PMA), or interleukin (IL)-18 for $15 \mathrm{~min}$. (D) Summary of frequencies of NF-kB(pS529)+ cells either treated with medium or IL-18 over time, $n=5$ donors. Symbols indicate mean and error bars SEM. Statistical analysis performed with repeated-measures two-way ANOVA with Bonferroni correction. (E,F) NK cells were cultured with K562/HLA-E in the presence of IL-12 + 18, IL-12, or IL-18. (E) Frequencies of $\Delta \mathrm{IFN}-\gamma^{+}$[calculated by subtraction of the frequency of interferon (IFN)- $\gamma^{+}$cells in the presence of K562/HLA-E alone] and (F) $\Delta \mathrm{TNF}^{+}$adaptive NK cells, $n=12$ donors. Symbols indicate mean and error bars SEM. Statistical analysis performed with Friedman and Dunn's multiple comparison test. (G,H) NK cells were cultured with K562/HLA-E and varying concentrations of IL-18. (G) Frequencies of $\Delta \mathrm{IFN}-\gamma^{+}$and (F) $\Delta \mathrm{TNF}^{+}$adaptive NK cells, $n=6$ donors. Symbols indicate mean and error bars SEM. Statistical analysis performed with Friedman and Dunn's multiple comparison test. NS, not significant; ${ }^{*} p<0.05,{ }^{* \star} p<0.01,{ }^{* \star *} p<0.001$, and ${ }^{* \star * *} p<0.0001$.

While previous studies have demonstrated that CD56 $6^{\text {dim }}$ but not CD $56^{\text {bright }} \mathrm{NK}$ cells benefit from addition of IL-12 + 18 during target-cell encounter (33), selective costimulation via IL-18 but not IL-12 is exclusive to adaptive NK cells and implies a pivotal role of IL-18 in modulating the functional response of this NK-cell subset. Notably, IL-18 was reported to synergize with low doses of common $\gamma$ chain cytokines IL-2, IL-15, and IL-21 in potentiating NK-cell activation (34), and the description of IL-1R8 as a negative regulator of the IL-18-IL-18R axis (35) highlights the prominent contribution of IL-18 to antiviral as well as antitumor NK-cell effector functions. Thus, IL-18 might act as a key indicator of inflammatory burden for adaptive NK cells and calibrate their cytokine production accordingly.

NKG2C expression marks the majority of adaptive NK-cell populations, but $\mathrm{HCMV}$-induced $\mathrm{NKG}^{-} \mathrm{C}^{-}$adaptive $\mathrm{NK}$ cells have been described in KLRC2 (encoding NKG2C)-sufficient and -deficient humans $(8,10,14,36)$. In agreement with these studies, NKG2C cross-linking was sufficient but not required to enable costimulation by exogenous cytokines, since CD16-dependent activation of adaptive NK cells by rituximab-coated 721.221 target cells similarly benefited from costimulation. Based on these data, we expect that other activating receptors, which can efficiently trigger IFN- $\gamma$ and TNF production of NKG2C- ${ }^{-}$adaptive NK cells, could be functionally calibrated by concomitant IL-18 signaling.

Although both EBV (37) and chronic hepatitis (38) were reported to not affect adaptive NK-cell expansions in HCMVseropositive individuals, provision of ample costimulatory signals-by cytokines and/or cellular ligands-could permit the activation of pre-existing HCMV-induced adaptive NK cells during heterologous infections including Hantavirus (39), HIV-1 $(8,40)$, or Chikungunya-virus infection $(41)$.

In summary, our study revealed that adaptive $\mathrm{NKG}_{2} \mathrm{C}^{+}$ NK cells can indeed be costimulated by IL-18 during target-cell encounter, resulting in augmented cytokine production. We propose that adaptive NK cells may act as a target cell-restricted inflammation-sensing immune hub, which is able to relay proinflammatory signals to bystander cells and thereby potentially amplify ensuing immune responses. 


\section{MATERIALS AND METHODS}

\section{Human Samples}

Buffy coats of HCMV-seropositive healthy blood donors were obtained from the German Red Cross as approved by Charité ethics commission (EA1/149/12). PBMCs were isolated by density gradient centrifugation (Ficoll Paque Plus, GE Healthcare) and screened for the presence of adaptive $\mathrm{NKG}_{2} \mathrm{C}^{+} \mathrm{NK}$ cells as previously described (42). In brief, coexpression analysis was employed to detect adaptive $\mathrm{CD} 2^{+} \mathrm{CD}^{+} 7^{+} \mathrm{ILT}_{2}{ }^{+}$Siglec-7 ${ }^{-}$ $\mathrm{NKp}^{-} 0^{-} \mathrm{NKG}_{2} \mathrm{~A}^{-}$within the $\mathrm{CD}^{\mathrm{dim}} \mathrm{NKG}^{+} \mathrm{C}^{+}$population (Figures S1A,B in Supplementary Material). Subsequently, CD56 ${ }^{+}$ cells were MACS-enriched (CD56 MicroBeads, Miltenyi Biotec) from donors containing adaptive NK-cell populations and cryopreserved in fetal bovine serum (FBS; Biowest) containing 10\% DMSO (Sigma). In the following assays, adaptive NK cells were gated as $\mathrm{CD}^{2} 6^{\mathrm{dim}} \mathrm{CD} 57^{+} \mathrm{NKG} 2 \mathrm{~A}^{-} \mathrm{NKG}^{-\mathrm{C}^{+}}$cells (Figure $\mathrm{S} 1 \mathrm{C}$ in Supplementary Material).

\section{Cells, Cell Lines, and HLA-E Surface Stabilization}

Primary HUVEC (Lonza) were maintained in endothelial growth medium (Lonza or PromoCell) containing Vascular Endothelial Growth Factor according to the manufacturer's instructions. 721.221 were maintained in complete medium [RPMI-1640 containing glutamine and supplemented with $10 \%(\mathrm{v} / \mathrm{v})$ FBS, $20 \mu \mathrm{M}$ $\beta$-mercaptoethanol, and $100 \mathrm{U} / \mathrm{mL}$ penicillin-streptomycin; all Thermo Fisher] and K562 transfected with HLA-E* ${ }^{\star}$ 1:03 [K562/ HLA-E; provided by E. Weiss (43)] were maintained in complete medium containing $1 \mathrm{mg} / \mathrm{mL}$ Geneticin (InVivogen). Prior to functional assays, surface expression of HLA-E by K562/HLA-E was stabilized by pulsing with $300 \mu \mathrm{M}$ synthetic $\mathrm{HLA}-\mathrm{Cw}^{\star} 01$ signal peptide VMAPRTLIL (Peptides\&Elephants) for 16-18 h in serum-free Opti-MEM (Thermo Fisher), similar to previous reports (44).

\section{Flow Cytometry}

Single cell suspensions were stained with different combinations of fluorochrome-conjugated antibodies (Table S1 in Supplementary Material), and dead cells were excluded using Fixable Dead Cell Stain Kit (Thermo Fisher) or Fixable Viability Kit (BioLegend). For intracellular staining of IFN- $\gamma$ and TNF, cells were surface stained and fixated with 2\% PFA (EMS Sciences), followed by treatment with Permeabilizing Solution 2 (BD Biosciences). Data were acquired on an LSR Fortessa (BD Biosciences) and analyzed with FlowJo vX (FlowJo LLC) software. ARIA or ARIA II instruments (both BD Biosciences) were used for cell sorting experiments.

\section{Cytokine Stimulations}

To assess IFN- $\gamma$ production upon stimulation with cytokines, $\mathrm{CD}^{2} 6^{+}$MACS-enriched cells were thawed, FACS-purified for viable $\mathrm{CD}^{-}{ }^{-} \mathrm{CD} 56^{+} \mathrm{NK}$ cells, and cultured in complete medium in the presence or absence of $10 \mathrm{ng} / \mathrm{mL}$ human recombinant IL-12 (Miltenyi Biotec) and $100 \mathrm{ng} / \mathrm{mL}$ IL-18 (MBL) for $24 \mathrm{~h}$. GolgiPlug and GoligStop (both BD Biosciences) were added according to the manufacturer's instructions and present during the last $5 \mathrm{~h}$ of the stimulation.

\section{Functional Assays and Cytokine Costimulation}

For functional assays, CD56 ${ }^{+}$MACS-enriched cells were thawed, FACS-purified for viable $\mathrm{CD}^{-} \mathrm{CD}^{-} 6^{+} \mathrm{NK}$ cells, and rested overnight in complete medium. The next day, $2 \times 10 \mathrm{e} 5 \mathrm{NK}$ cells were mixed with $1 \times 10 \mathrm{e} 5$ target cells in V-bottom 96 -well plates and the indicated concentrations of IL-12 and/or IL-18 were added. For cross-linking of CD16, rituximab (Roche) was present during the assay at the indicated concentrations. For sole engagement of NKG2C, MACSi beads (Miltenyi) were coated with an agonistic anti-NKG2C antibody (R\&D Systems; biotinylated in-house) as previously described (11), and $1 \times 10 \mathrm{e} 6 \alpha \mathrm{NKG} 2 \mathrm{C}$ beads were added to $2 \times 10 \mathrm{e} 5 \mathrm{NK}$ cells. After $1 \mathrm{~h}$ incubation, GolgiPlug and GolgiStop (both BD Biosciences) were added according to the manufacturer's instructions, and the assay continued for $5 \mathrm{~h}$.

\section{Protein Measurements and Generation of Conditioned Medium}

To measure protein concentrations in supernatants, CD56 ${ }^{+}$ MACS-enriched cells were thawed, FACS-purified for viable $\mathrm{CD}^{-} \mathrm{CD}^{-\mathrm{dim}} \mathrm{CD}^{-} 7^{+} \mathrm{NKG}^{+} \mathrm{C}^{+}$adaptive $\mathrm{NK}$ cells, and rested overnight in complete medium. The next day, $2 \times 10 \mathrm{e} 5$ adaptive NK cells were mixed with $1 \times 10 \mathrm{e} 5 \mathrm{~K} 562 / \mathrm{HLA}-\mathrm{E}$ target cells in V-bottom 96-well plates in the absence or presence of IL-12 + 18 . After $6 \mathrm{~h}$ of culture, cell-free supernatant was obtained by repeated centrifugation and stored at $-80^{\circ} \mathrm{C}$. IFN- $\gamma$ and TNF protein levels were measured using LegendPlex (BioLegend) according to the manufacturer's instructions.

Conditioned medium was obtained by mixing thawed supernatants 1:4 with EGM-2 for direct use in bystander cell assays.

\section{Bystander Cell Assays}

For bystander cell assays, $2.5 \times 10 \mathrm{e} 4 \mathrm{HUVEC}$ were allowed to adhere in 48-well plates in EGM-2, followed by treatment with conditioned medium in the presence of $10 \mu \mathrm{g} / \mathrm{mL}$ blocking anti-IFN- $\gamma$ (clone B27) and anti-TNF (clone Mab1) or IgG1 isotype control (clone MPOC-21; all BioLegend) antibodies. When indicated, HUVEC were directly treated with $10 \mathrm{ng} / \mathrm{mL}$ IL-12 and $100 \mathrm{ng} / \mathrm{mL}$ IL-18, $1 \mathrm{ng} / \mathrm{mL}$ IFN- $\gamma$ (R\&D Systems), or $1 \mathrm{ng} / \mathrm{mL}$ TNF (kindly provided by AA Kruglov). To determine HLA class I expression, HUVEC were recovered from plates by trypsinization at $37^{\circ} \mathrm{C}$ after $40 \mathrm{~h}$ of treatment with conditioned medium, followed by FACS staining. Analysis of adhesion molecule expression by FACS was performed after $6 \mathrm{~h}$ incubation with conditioned medium and recovery of HUVEC using Accutase (Sigma) at room temperature. For assessing transcript levels, HUVEC were lysed after $24 \mathrm{~h}$ of treatment, and total RNA was isolated using RNeasy Micro Plus Kit (Qiagen) according to the manufacturer's instructions.

\section{Ex Vivo Sorting of NK- and T-Cell Subsets}

For ex vivo sorting experiments, $\mathrm{NK}$ and $\mathrm{T}$ cells were enriched from freshly isolated PBMC using CD56 Microbeads and CD8 
Microbeads (both Miltenyi), respectively. MACS-enriched cells were sorted to $>95 \%$ purity as immature conventional (CD3 ${ }^{-}$ $\mathrm{CD}^{2} 6^{\mathrm{dim}} \mathrm{CD}^{-} 7^{-} \mathrm{NKG}^{-}$) NK cells, mature conventional (CD3 ${ }^{-}$ $\mathrm{CD}^{\text {dim }} \mathrm{CD}^{\mathrm{d}} 7^{+} \mathrm{NKG}^{-} \mathrm{C}^{-}$) $\mathrm{NK}$ cells, adaptive $\left(\mathrm{CD}^{-} \mathrm{CD}^{-\mathrm{dim}}\right.$ $\left.\mathrm{CD} 7^{+} \mathrm{NKG}_{2} \mathrm{C}^{+}\right) \mathrm{NK}$ cells, naïve $\left(\mathrm{CD}^{+} \mathrm{CD}^{-} \mathrm{CD}^{+} \mathrm{CD}^{+} 5 \mathrm{RA}^{+}\right.$

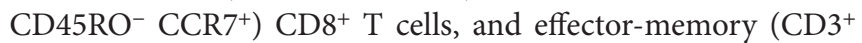
$\left.\mathrm{CD}^{-} \mathrm{CD}^{+} \mathrm{CD}^{-} 5 \mathrm{RA}^{-} \mathrm{CD}^{-} 5 \mathrm{RO}^{+} \mathrm{CCR}^{-}\right) \mathrm{CD}^{+} \mathrm{T}$ cells. Total RNA was isolated using the AllPrep DNA/RNA Mini Kit (Qiagen) according to the manufacturer's instructions.

\section{Quantitative RT-PCR}

To determine transcript levels, 50 ng of total RNA was reverse transcribed using TaqMan Reverse Transcription Reagents (Applied Biosciences), and quantitative real-time PCR was performed with TaqMan gene expression assays (Table S2 in Supplementary Material) on a StepOnePlus instrument (Applied Biosciences).

\section{Signaling Assays}

To investigate phosphorylation of NF- $\kappa \mathrm{B}$ and STAT4, CD56 ${ }^{+}$ MACS-enriched cells were thawed, FACS-purified for viable $\mathrm{CD}^{-} \mathrm{CD}^{-\mathrm{dim}} \mathrm{CD}^{-} 7^{+} \mathrm{NKG}^{+} \mathrm{C}^{+}$adaptive $\mathrm{NK}$ cells, CD56 ${ }^{\text {dim }}$ $\mathrm{CD}^{2} 7^{-}$immature $\mathrm{NK}$ cells, or $\mathrm{CD} 56^{\mathrm{dim}} \mathrm{CD} 57^{+}$mature NK cells, and rested overnight in complete medium. The next day, $0.25-1 \times 10 \mathrm{e} 5 \mathrm{NK}$ cells were either treated with complete medium, $50 \mathrm{ng} / \mathrm{mL}$ phorbol-12-myristat-13-acetat, $100 \mathrm{ng} / \mathrm{mL}$ IL-18, or $100 \mathrm{ng} / \mathrm{mL}$ IL-12 for the indicated time periods and directly fixated in Cytofix (BD Biosciences). Fixated cells were permeabilized in PermIII (BD Biosciences) on ice, followed by staining of NF- $\mathrm{B}$ (pS529) or STAT4 (pY693) (Table S1 in Supplementary Material).

\section{Statistical Analysis}

Data were analyzed using GraphPad Prism 7. Wilcoxon matchedpairs test was applied to compare two groups of paired samples, while Friedman and Dunn's multiple comparison test was used to compare three or more paired groups. When evaluating paired data sets with two variables (e.g., treatment and time),

\section{REFERENCES}

1. Biron CA, Byron KS, Sullivan JL. Severe herpes virus infections in an adolescent without natural killer cells. NEngl J Med (1989) 320:1731-5. doi:10.1056/ NEJM198906293202605

2. Annunziato F, Romagnani C, Romagnani S. The 3 major types of innate and adaptive cell-mediated effector immunity. J Allergy Clin Immunol (2015) 135:626-35. doi:10.1016/j.jaci.2014.11.001

3. Cooper MA, Elliott JM, Keyel PA, Yang L, Carrero JA, Yokoyama WM. Cytokine-induced memory-like natural killer cells. Proc Natl Acad Sci U S A (2009) 106:1915-9. doi:10.1073/pnas.0813192106

4. Romee R, Schneider SE, Leong JW, Chase JM, Keppel CR, Sullivan RP, et al. Cytokine activation induces human memory-like NK cells. Blood (2012) 120:4751-60. doi:10.1182/blood-2012-04-419283

5. O’Leary JG, Goodarzi M, Drayton DL, Von Andrian UH. T cell- and B cell-independent adaptive immunity mediated by natural killer cells. Nat Immunol (2006) 7:507-16. doi:10.1038/ni1332

6. Sun JC, Beilke JN, Lanier LL. Adaptive immune features of natural killer cells. Nature (2009) 457:557-61. doi:10.1038/nature07665 repeated-measures two-way ANOVA with Bonferroni correction was employed to determine statistical significance at each time point. NS indicates not significant, ${ }^{*} p<0.05,{ }^{* *} p<0.01$, ${ }^{* * *} p<0.001$, and ${ }^{* * *} p<0.0001$.

\section{ETHICS STATEMENT}

Buffy coats of HCMV-seropositive healthy blood donors were obtained from the German Red Cross as approved by Charité ethics commission (EA1/149/12).

\section{AUTHOR CONTRIBUTIONS}

QH conceived, designed, and administrated the study. QH, TR, and JD performed experiments and analyzed data. QH and CR interpreted data and drafted the manuscript. CR supervised the work. All the authors read and approved the submitted version of the manuscript.

\section{ACKNOWLEDGMENTS}

We thank the DRFZ FCCF for cell sorting, U Uhlig for technical assistance, and DC Hernández for critical reading of the manuscript. AA Kruglov and P Saikali provided insightful comments on experimental procedures and during manuscript preparation. This work was supported by the Leibniz ScienceCampus Chronic Inflammation (www.chronische-entzuendung.org) as well as German Research Foundation (DFG) grants SFB 650, RO3565/2-1, and RO3565/4-1 to CR; CR and QH are supported by the DFG Heisenberg Program (RO 3565/1-1) and the Leibniz Association (Leibniz Graduate School for Rheumatology), respectively.

\section{SUPPLEMENTARY MATERIAL}

The Supplementary Material for this article can be found online at http://www.frontiersin.org/articles/10.3389/fimmu.2017.01976/ full\#supplementary-material.

7. Reeves RK, Li H, Jost S, Blass E, Li H, Schafer JL, et al. Antigen-specific NK cell memory in rhesus macaques. Nat Immunol (2015) 16:927-32. doi:10.1038/ ni. 3227

8. Guma M, Cabrera C, Erkizia I, Bofill M, Clotet B, Ruiz L, et al. Human cytomegalovirus infection is associated with increased proportions of NK cells that express the CD94/NKG2C receptor in aviremic HIV-1-positive patients. J Infect Dis (2006) 194:38-41. doi:10.1086/504719

9. Beziat V, Dalgard O, Asselah T, Halfon P, Bedossa P, Boudifa A, et al. CMV drives clonal expansion of NKG2C+ NK cells expressing self-specific KIRs in chronic hepatitis patients. Eur J Immunol (2012) 42:447-57. doi:10.1002/ eji.201141826

10. Beziat V, Liu LL, Malmberg JA, Ivarsson MA, Sohlberg E, Bjorklund AT, et al. NK cell responses to cytomegalovirus infection lead to stable imprints in the human KIR repertoire and involve activating KIRs. Blood (2013) 121:2678-88. doi:10.1182/blood-2012-10-459545

11. Luetke-Eversloh M, Hammer Q, Durek P, Nordstrom K, Gasparoni G, Pink M, et al. Human cytomegalovirus drives epigenetic imprinting of the IFNG locus in NKG2Chi natural killer cells. PLoS Pathog (2014) 10:e1004441. doi:10.1371/ journal.ppat.1004441 
12. Schlums H, Cichocki F, Tesi B, Theorell J, Beziat V, Holmes TD, et al. Cytomegalovirus infection drives adaptive epigenetic diversification of NK cells with altered signaling and effector function. Immunity (2015) 42:443-56. doi:10.1016/j.immuni.2015.02.008

13. Hammer Q, Romagnani C. About training and memory: NK-cell adaptation to viral infections. Adv Immunol (2016) 133:171-207. doi:10.1016/ bs.ai.2016.10.001

14. Liu LL, Landskron J, Ask EH, Enqvist M, Sohlberg E, Traherne JA, et al. Critical role of $\mathrm{CD} 2$ co-stimulation in adaptive natural killer cell responses revealed in NKG2C-deficient humans. Cell Rep (2016) 15:1088-99. doi:10.1016/j. celrep.2016.04.005

15. Hayden FG, Fritz R, Lobo MC, Alvord W, Strober W, Straus SE. Local and systemic cytokine responses during experimental human influenza A virus infection. Relation to symptom formation and host defense. JClin Invest (1998) 101:643-9. doi:10.1172/JCI1355

16. Brennan K, Bowie AG. Activation of host pattern recognition receptors by viruses. Curr Opin Microbiol (2010) 13:503-7. doi:10.1016/j.mib.2010.05.007

17. Hermesh T, Moltedo B, Moran TM, Lopez CB. Antiviral instruction of bone marrow leukocytes during respiratory viral infections. Cell Host Microbe (2010) 7:343-53. doi:10.1016/j.chom.2010.04.006

18. Schroder K, Hertzog PJ, Ravasi T, Hume DA. Interferon-gamma: an overview of signals, mechanisms and functions. J Leukoc Biol (2004) 75:163-89. doi:10.1189/jlb.0603252

19. Mai J, Virtue A, Shen J, Wang H, Yang XF. An evolving new paradigm: endothelial cells - conditional innate immune cells. J Hematol Oncol (2013) 6:61. doi:10.1186/1756-8722-6-61

20. Carman CV, Martinelli R. T lymphocyte-endothelial interactions: emerging understanding of trafficking and antigen-specific immunity. Front Immunol (2015) 6:603. doi:10.3389/fimmu.2015.00603

21. Kalliolias GD, Ivashkiv LB. TNF biology, pathogenic mechanisms and emerging therapeutic strategies. Nat Rev Rheumatol (2016) 12:49-62. doi:10.1038/ nrrheum.2015.169

22. Lertmemongkolchai G, Cai G, Hunter CA, Bancroft GJ. Bystander activation of CD8+ $\mathrm{T}$ cells contributes to the rapid production of IFN-gamma in response to bacterial pathogens. J Immunol (2001) 166:1097-105. doi:10.4049/ jimmunol.166.2.1097

23. Berg RE, Crossley E, Murray S, Forman J. Memory CD $8+\mathrm{T}$ cells provide innate immune protection against Listeria monocytogenes in the absence of cognate antigen. J Exp Med (2003) 198:1583-93. doi:10.1084/jem.20031051

24. Kambayashi T, Assarsson E, Lukacher AE, Ljunggren HG, Jensen PE. Memory CD8+ T cells provide an early source of IFN-gamma. J Immunol (2003) 170:2399-408. doi:10.4049/jimmunol.170.5.2399

25. Sun JC, Madera S, Bezman NA, Beilke JN, Kaplan MH, Lanier LL. Proinflammatory cytokine signaling required for the generation of natural killer cell memory. J Exp Med (2012) 209:947-54. doi:10.1084/jem. 20111760

26. Min-Oo G, Lanier LL. Cytomegalovirus generates long-lived antigen-specific NK cells with diminished bystander activation to heterologous infection. J Exp Med (2014) 211:2669-80. doi:10.1084/jem.20141172

27. Gee K, Guzzo C, Che Mat NF, Ma W, Kumar A. The IL-12 family of cytokines in infection, inflammation and autoimmune disorders. Inflamm Allergy Drug Targets (2009) 8:40-52. doi:10.2174/187152809787582507

28. Novick D, Kim S, Kaplanski G, Dinarello CA. Interleukin-18, more than a Th1 cytokine. Semin Immunol (2013) 25:439-48. doi:10.1016/j.smim.2013.10.014

29. Ross ME, Caligiuri MA. Cytokine-induced apoptosis of human natural killer cells identifies a novel mechanism to regulate the innate immune response. Blood (1997) 89:910-8.

30. Corat MA, Schlums H, Wu C, Theorell J, Espinoza DA, Sellers SE, et al. Acquired somatic mutations in PNH reveal long-term maintenance of adaptive NK cells independent of HSPCs. Blood (2017) 129:1940-6. doi:10.1182/ blood-2016-08-734285

31. Schlums H, Jung M, Han H, Theorell J, Bigley V, Chiang SC, et al. Adaptive NK cells can persist in patients with GATA2 mutation depleted of stem and progenitor cells. Blood (2017) 129:1927-39. doi:10.1182/ blood-2016-08-734236

32. van de Berg PJ, Heutinck KM, Raabe R, Minnee RC, Young SL, Van DonselaarVan Der Pant KA, et al. Human cytomegalovirus induces systemic immune activation characterized by a type 1 cytokine signature. J Infect Dis (2010) 202:690-9. doi:10.1086/655472

33. Fauriat C, Long EO, Ljunggren HG, Bryceson YT. Regulation of human NK-cell cytokine and chemokine production by target cell recognition. Blood (2010) 115:2167-76. doi:10.1182/blood-2009-08-238469

34. Nielsen CM, Wolf AS, Goodier MR, Riley EM. Synergy between common gamma chain family cytokines and IL-18 potentiates innate and adaptive pathways of NK Cell Activation. Front Immunol (2016) 7:101. doi:10.3389/ fimmu.2016.00101

35. Molgora M, Bonavita E, Ponzetta A, Riva F, Barbagallo M, Jaillon S, et al. IL$1 \mathrm{R} 8$ is a checkpoint in NK cells regulating anti-tumour and anti-viral activity. Nature (2017) 551:110-4. doi:10.1038/nature24293

36. Della Chiesa M, Falco M, Bertaina A, Muccio L, Alicata C, Frassoni F, et al. Human cytomegalovirus infection promotes rapid maturation of NK cells expressing activating killer Ig-like receptor in patients transplanted with NKG2C-/- umbilical cord blood. J Immunol (2014) 192:1471-9. doi:10.4049/ jimmunol.1302053

37. Hendricks DW, Balfour HH Jr, Dunmire SK, Schmeling DO, Hogquist KA, Lanier LL. Cutting edge: NKG2C(hi)CD57+ NK cells respond specifically to acute infection with cytomegalovirus and not Epstein-Barr virus. J Immunol (2014) 192:4492-6. doi:10.4049/jimmunol.1303211

38. Malone DFG, Lunemann S, Hengst J, Ljunggren HG, Manns MP, Sandberg JK, et al. Cytomegalovirus-driven adaptive-like natural killer cell expansions are unaffected by concurrent chronic hepatitis virus infections. Front Immunol (2017) 8:525. doi:10.3389/fimmu.2017.00525

39. Bjorkstrom NK, Lindgren T, Stoltz M, Fauriat C, Braun M, Evander M, et al. Rapid expansion and long-term persistence of elevated NK cell numbers in humans infected with hantavirus. J Exp Med (2011) 208:13-21. doi:10.1084/ jem.20100762

40. Brunetta E, Fogli M, Varchetta S, Bozzo L, Hudspeth KL, Marcenaro E, et al. Chronic HIV-1 viremia reverses NKG2A/NKG2C ratio on natural killer cells in patients with human cytomegalovirus co-infection. AIDS (2010) 24:27-34. doi:10.1097/QAD.0b013e3283328d1f

41. Petitdemange C, Becquart P, Wauquier N, Beziat V, Debre P, Leroy EM, et al. Unconventional repertoire profile is imprinted during acute chikungunya infection for natural killer cells polarization toward cytotoxicity. PLoS Pathog (2011) 7:e1002268. doi:10.1371/journal.ppat.1002268

42. Hammer Q, Romagnani C. OMIP-039: detection and analysis of human adaptive NKG2C+ natural killer cells. Cytometry A (2017) 91(10):997-1000. doi:10.1002/cyto.a.23168

43. Ulbrecht M, Martinozzi S, Grzeschik M, Hengel H, Ellwart JW, Pla M, et al. Cutting edge: the human cytomegalovirus UL40 gene product contains a ligand for HLA-E and prevents NK cell-mediated lysis. J Immunol (2000) 164:5019-22. doi:10.4049/jimmunol.164.10.5019

44. Heatley SL, Pietra G, Lin J, Widjaja JM, Harpur CM, Lester S, et al. Polymorphism in human cytomegalovirus UL40 impacts on recognition of human leukocyte antigen-E (HLA-E) by natural killer cells. J Biol Chem (2013) 288:8679-90. doi:10.1074/jbc.M112.409672

Conflict of Interest Statement: The authors declare that the research was conducted in the absence of any commercial or financial relationships that could be construed as a potential conflict of interest.

Copyright (c) 2018 Hammer, Rückert, Dunst and Romagnani. This is an open-access article distributed under the terms of the Creative Commons Attribution License (CC BY). The use, distribution or reproduction in other forums is permitted, provided the original author(s) or licensor are credited and that the original publication in this journal is cited, in accordance with accepted academic practice. No use, distribution or reproduction is permitted which does not comply with these terms. 